\title{
EINZELAUSGABE
}

\section{DAS ERZBISTUM MAGDEBURG UND DIE ERSTE ORGANISATION DER CHRISTLICHEN KIRCHE IN POLEN}

VON

\author{
P. KEHR
}

AUS DEN ABHANDLUNGEN DER PREUSSISCHEN AKADEMIE DER WISSENSCHAFTEN JAHRGANG 1920. PHIL.-HIST. KLASSE. Nв. 1

\section{BERLIN 1920}

I'RIAG DER AKADEMIE DER WISSENSCHAFTEN

IN KOMMISSION BEI DER VEREINIGUNG WISSENSCHAFTLICHER VERLEGER WALTER DE GRUYTER U. CO VORMAIS G. J. GÖSCHEN'SCHE VERLAGSHANDLUNG. J. GUTTENTAG, VERLAGSBUCHHANDLUNG. GEORG REIMER. KARL. J. TRÛBNER. VEIT U. COMP. 
Gelesen in der Sitzung der phil.-hist. Klasse am 29. November 1919.

Zum Druck eingereicht an 15. April 1920, ausgegeben am 24. Juni 1920. 